\title{
The Molecular Mechanism of Sirt1 Signaling Pathway in Brain Injury of Newborn Rats Exposed to Hyperoxia
}

\author{
Lan Kang, ${ }^{a, b}$ Wenbin Dong, ${ }^{b}$ Ying Ruan, ${ }^{b}$ Rong Zhang, ${ }^{b}$ and Xingyong Wang*,a \\ a Department of Pediatric Research Institute, Children's Hospital of Chongqing Medical University; Chongqing \\ 400014, China: and ${ }^{b}$ Department of Newborn Medicine, The Affiliated Hospital of Southwest Medical University; \\ Luzhou 646000, Sichuan, China. \\ Received May 9, 2019; accepted July 30, 2019; advance publication released online September 13,2019
}

The aim of the study was to investigate the changes in the reactive oxygen species (ROS), Sirt1, p53 and acetylated $\mathbf{p 3}$ in brain tissue of newborn rats exposed to hyperoxia to clarify the role of Sirt1 signaling pathway in brain injury. Neonate rats were randomly divided into normoxic group and hyperoxic group. Rats in the normoxic group were exposed to room air while the rats in the hyperoxic group were put in a hyperoxic chamber ( $80 \pm 5 \%$ oxygen) for 1 to $14 \mathrm{~d}$. Data, including weight growth, the water content of brain tissue, hematoxyline and eosin (H\&E) and terminal deoxynucleotidyl transferase-mediated deoxyuridine triphosphate nick-end labeling (Tunel) stain, ROS expression, the relative expression of Sirt1 mRNA and p53 mRNA, and the protein relative expression of Sirt1, p53 and acetylated p53 were analyzed at 1, 7 and $14 \mathrm{~d}$ after exposure. A reduced body weight and increased water content were observed in the brain tissue of hyperoxic group compared to normoxic group. HE staining and Tunel staining of brain tissue suggested that cell damaged after hyperoxic exposure. RT-PCR and Western blot results showed that the expression of Sirt1 in the hyperoxic group was lower than that in the normoxic group while the expression of p53 was higher than that in the normoxic group. In addition, Western blot data indicated acetylated p53 expression was higher in the hyperoxic group. Hyperoxic exposure can lead to brain injury in newborn Sprague-Dawley (SD) rats. These events might be regulated by the Sirt1 pathway, which downregulated the deacetylation of p53.

Key words Sirt1; hyperoxia; brain injury

\section{INTRODUCTION}

Thanks to advancements in medical technology, the birth rate and survival rate of premature babies are constantly increasing. It has been estimated that some 15 million babies are prematurely born every year, ${ }^{1)}$ which has also led to an increase in the recognition of brain injuries due to preterm delivery. So far, many factors have been associated with brain injury, including hyperoxia. ${ }^{2)}$ Premature infants often develop hypoxemia in the early stage of life, especially when accompanied with pulmonary disease or cardiovascular disease. Atmospheric concentrations of oxygen therapy can significantly improve neonatal hypoxic condition; therefore, oxygen has become an important treatment approach for these patients. Nevertheless, high oxygen ${ }^{3)}$ can stimulate the production of a large number of active oxygen substances. Infants, especially the premature infants, have an undeveloped immune and antioxidant defense system, and thus are more vulnerable to these substances.

Silent information regulator family protein (sirtuins, SIRTs) is a kind of histone deacetylation enzyme dependent on nicotinamide adenine dinucleotide (NAD +). ${ }^{4}$ The Sirt1, which is highly expressed in human tissues, is involved in deacetylation of histone lysine (Lys) residues and non-histone Lys residues, and can adjust the physiological function of the target protein. Studies have shown that many proteins regulating cell proliferation and apoptosis work as a substrates for a Sirt1, such as p53. ${ }^{5)}$ Existing studies have confirmed that Sirt1 can bind and deacetylate p53, negatively regulate the transcriptional activation of p53-mediated downstream apoptosis induction factors, and inhibit apoptosis. The aim of this study was to investigate the changes in the reactive oxygen species (ROS), Sirt1, p53 and acetylated-p53 (AC-p53) in brain tissue of newborn rats exposed to hyperoxia, in order to clarify the role of Sirt1 signaling pathway in brain injury.

\section{MATERIALS AND METHODS}

Animals Sprague-Dawley (SD) newborn rats, weighing $5.4-8.6 \mathrm{~g}$, were provided by the experimental animal center of the Southwest Medical University. All the animals were housed in an environment with temperature of $22 \pm 1^{\circ} \mathrm{C}$, relative humidity of $50 \pm 1 \%$ and a light/dark cycle of $12 / 12 \mathrm{~h}$. All animal studies (including the mice euthanasia procedure) were done in compliance with the regulations and guidelines of the Southwest Medical University institutional animal care and conducted according to the AAALAC and the IACUC guidelines (License number of experimental animal: SYXK (Sichuan) 2018-065).

A total of 108 newborn SD rats (24h old rats) were randomly divided into normoxic group and hyperoxic group. Brain tissues were obtained after exposing the rats to hyperoxia for 1,7 and $14 \mathrm{~d}$, respectively. Consequently, each group was divided into three subgroups (18 rats/group): normoxic 1d, normoxic $7 \mathrm{~d}$, normoxic $14 \mathrm{~d}$; hyperoxic $1 \mathrm{~d}$, hyperoxic $7 \mathrm{~d}$ and hyperoxic $14 \mathrm{~d}$.

High-oxygen brain injury model was established by highconcentration oxygen induction. ${ }^{6}$ ) In the normoxic group, newborn rats and lactating mothers were exposed to normal temperature and pressure air and were free to suckle and for- 
age. In hyperoxic group, rats and their breastfeeding mothers were put in a hyperoxic chamber $(80 \pm 5 \%$ oxygen $)$ for 1 to $14 \mathrm{~d}$. The soda lime was placed in the chamber to absorb excess carbon dioxide and water. The indoor temperature was kept at $22 \pm 1{ }^{\circ} \mathrm{C}$ and the relative humidity was at $50 \pm 1 \%$. The breastfeeding mothers in normoxic and hyperoxic groups were exchanged every $24 \mathrm{~h}$ in order to avoid decreasing lactation ability caused by stress reaction to oxygen poisoning.

Animal Growth The growth and general health conditions, including weight, reaction, skin color, foraging ability and survival rate were recorded on a daily basis. Weight gain was expressed by weight growth rate, and was calculated by the following formula: weight growth rate $=$ (weight at the time of sampling-birth weight)/birth weight $\times 100 \%$. The birth weight of newborn SD rats was measured by electronic scale within $2 \mathrm{~h}$ after birth. The body weight at the time of sampling was measured at days 1,7 and 14 after hyperoxic exposure.

Sample Preparation Rats were euthanized by inhalation ether anesthesia. The brain tissues were then collected after exposing the skull along the midline line of the head. Six brain tissues were obtained in each group in order to measure the water content. Additional six brain tissues were fixed in $4 \%$ paraformaldehyde, embed in paraffin and stained with hematoxylin and eosin (H\&E) and terminal deoxynucleotidyl transferase-mediated deoxyuridine triphosphate nick-end labeling (Tunel) staining. The remaining tissues were immediately snap frozen in liquid nitrogen and were then transferred to $-80^{\circ} \mathrm{C}$ for further analysis (reactive oxygen species, RTPCR and Western blot).

Determination of Water Content in Brain Tissue The brain tissue was weighed immediately after collection. Samples were recorded as the wet weight of the brain tissues. Consequently, the brain tissue was transferred into the $60^{\circ} \mathrm{C}$ drying oven to dry out the water; the tissue was weighted on a daily basis for three consecutive days until no weight decrease was observed, and the dry weight was achieved. The water content was measured using the following formula: $\mathrm{WC}=[($ wet weight - dry weight $) /$ wet weight $] \times 100 \%$.

Pathological Observation of Brain Tissue The brain tissues in each group were completely removed and fixed in $4 \%$ paraformaldehyde. After conventional washing, dehydration and paraffin implantation, paraffin sections with a thickness of 3-5um were stained with H\&E to observe the cell morphology and damage degree in each group.

Detection of Apoptosis in Brain Tissue In strict accordance with nuclear staining combined with Tunel method, pretreatment was performed on the brain tissue embedded in the sections, and nuclear staining was performed using the In Situ Cell Death Detection Kit (Roche, Switzerland). The nucleus color of apoptotic cell was brownish yellow. Apoptosis index $=($ the number of apoptotic cells/the total number of cells) $\times 100 \%$. Four Tunel staining sections were selected from each brain tissue in each group and five different fields were selected from each section under the light microscope (CX31, Olympus, Tokyo, Japan). The average apoptosis index was calculated by Image J software.

Detection of Brain Tissue Reactive Oxygen Species Reactive oxygen species were measured using the Tissue ROS Assay Kit (Genmed Scientifics Inc., U.S.A.). Brain tissues were homogenated on ice. Consequently, the OD (excitation light was $490 \mathrm{~nm}$, emission light was $520 \mathrm{~nm}$, the intensity was 800 ) and the protein concentration of the same sample were analyzed using the BCA method. The reactive oxygen concentration was calculated using the following formula: reactive oxygen concentration $=$ reactive oxygen $\mathrm{OD}$ value/protein concentration.

RNA Isolation and Quantitative (q) Real-Time PCR The total RNA was extracted using TRIzol method (Cwbiotech, Jiangsu, China). Consequently, $250 \mathrm{ng}$ of the RNA was reverse transcribed into cDNA (Cwbiotech, Jiangsu, China). Based on cDNA template for PCR amplification, and PCR reaction system for $20 \mu \mathrm{L}$ (RR820A, TaKaRa, Japan), PCR primers sequences were designed and synthesized by Sangon Biological Engineering (Shanghai) Co., LTD. The sequences of the primer pairs were as follows: $\beta$-actin (F: 5'-CCC ATC TAT GAGGGT TAC GC-3', R: 5' - TTT AAT GTC ACGCAC GAT TTC-3'); Sirt1 (F: 5'-GAT ACC TTGGAGCAGGTT GC3', R:5'-CCA CGA ACA GCT TCA CAA TC-3'); p53 (F: 5'-GTA CCG TAT GAG CCA CCT GAG-3'， R： 5'-TCC AGC GTG ATG ATGGTAAG-3'). The first step reaction conditions were: $95^{\circ} \mathrm{C}$ for $30 \mathrm{~s}$; the second step conditions were: $95^{\circ} \mathrm{C}$ for $5 \mathrm{~s}$, $60^{\circ} \mathrm{C}$ for $15 \mathrm{~s}, 72^{\circ} \mathrm{C}$ for $45 \mathrm{~s}, 40$ cycles; the third step conditions were: $95^{\circ} \mathrm{C}$ for $15 \mathrm{~s}, 1 \mathrm{~min} 60^{\circ} \mathrm{C}, 95^{\circ} \mathrm{C}$ for $15 \mathrm{~s}$. Quantitative real-time PCR was performed by the StepOnePlus RealTime PCR System (Applied Biosystems, U.S.A.).

The expressions of Sirt1 and p53 gene transcript were determined by relative quantitative method $(2-\Delta \Delta \mathrm{Ct})$ which took the $\beta$-actin in the normoxic group as an endogenous control for normalization of expression levels.

Western Blot The total protein was extracted and the protein concentration was determined using BCA method. After sodium dodecyl sulfate (SDS)-polyacrylamidogel electrophoresis (PAGE), the protein was separated and transferred to the polyvinylidene difluoride (PVDF) membrane protein. Antibodies were closed in skimmed milk powder, and were respectively incubated with Sirt1 antibody (sc-74504, Santa Cruz Biotechnology, Santa Cruz, CA, U.S.A.) (1:500), p53 antibody (ab131442, Abcam, Cambridge, U.K.) (1:1000), AC-p53 antibody (ab183544, Abcam) (1:1000), glyceraldehyde-3-phosphate dehydrogenase (GAPDH) antibody (10494-1-AP, Proteintech Group, Inc., U.S.A.) $(1: 14000)$ at $4^{\circ} \mathrm{C}$, using shaking table for one night. GAPDH was used as an internal control. After the PVDF membrane washing within PBST, the protein samples were incubated with HRP labeled Goat Anti Rabbit immunoglobulin G (IgG) (bs-0295G-HRP, Bioss Biotechnology Co., Ltd., China) $(1: 2000)$ at $37^{\circ} \mathrm{C}$ for $1 \mathrm{~h}$, and then were measured by ECL chemiluminescence method. The gel analysis imaging system was used for scanning and analysis. The absorbance (A) value of the protein bands was determined by the image analysis software, and the relative expression level of the target protein was expressed by the A target protein /AGapdh ratio.

Statistical Analysis The experimental data were processed by Graphpad prism5.0 software and analyzed by SPSS 17.0 statistical software. The measurement data were expressed as mean \pm standard deviation (S.D.). Least-significant difference (LSD) test was used for the comparison between the groups. $p<0.05$ was considered statistically significant. 


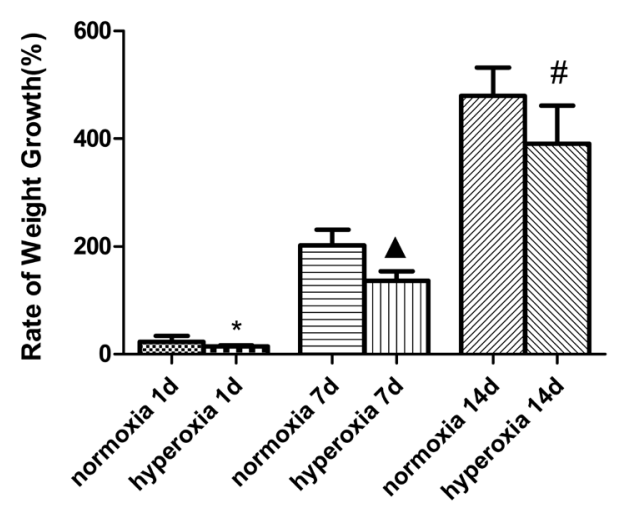

a

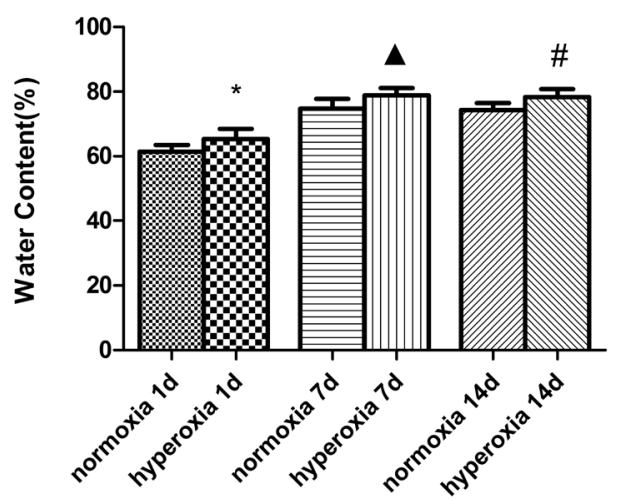

b

Fig. 1. (a): Body Weight Growth Rate at 1, 7 and 14 d in Each Group; (b): Changes in Brain Tissue Moisture Content in Each Group

(*Compared with normoxia 1d, $\boldsymbol{\Delta}$ Compared with normoxia 7d, \# Compared with normoxia 14d, $p<0.05$ ).

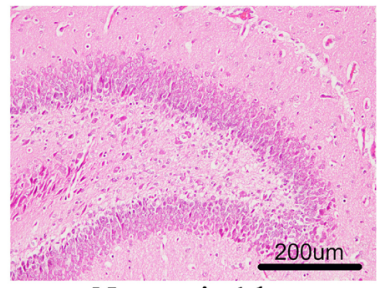

Normoxia 1d

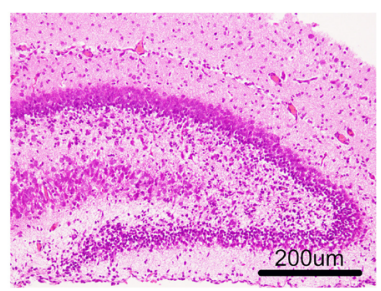

Hyperoxia 1d

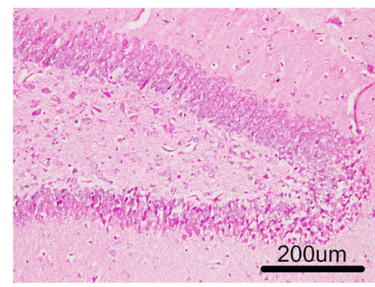

Normoxia 7d

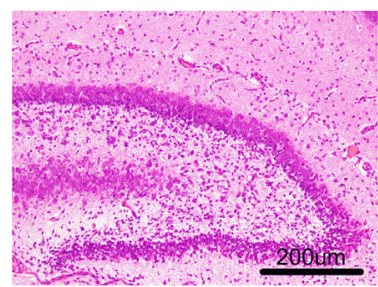

Hyperoxia 7d

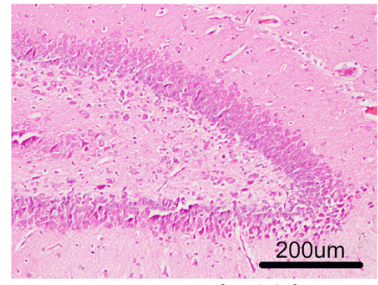

Normoxia 14d

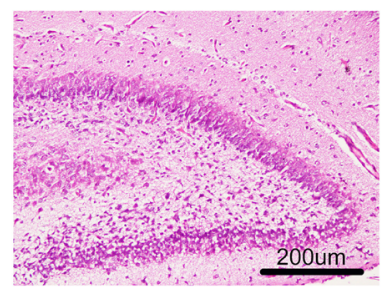

Hyperoxia 14d

Fig. 2. Brain Histopathological Changes Were Observed on Day 1, Day 7 and Day 14 in the Normoxic Group and the Hyperoxic Group (H\&E $\times 200$ ) (Color figure can be accessed in the online version.)

\section{RESULTS}

Hyperoxic Exposure Significantly Reduced the Rats Health Conditions Significant differences in animal growth and animal health conditions were observed in the hyperoxic group and the normoxic group. The newborn rats in normoxic group had good mental state, hyperactivity, sensitive reaction, ruddy skin and good growth; while the rats in the hyperoxic group gradually showed mental deterioration, decreased foraging, decreased activity, excessive ruddy skin, decreased body size, and even nervous system damage, such as head-shaking, gait instability, and convulsion, with the prolonged exposure to high-concentration oxygen environment. As shown in Fig. 1a, one day after hyperoxic exposure, the body weight growth rate of the hyperoxic group was significantly lower than that of the normoxic group $(14.15 \pm 2.06$ vs. $22.90 \pm 10.85$, respectively; $p<0.05$ ); after $7 \mathrm{~d}$ and $14 \mathrm{~d}$, the observed difference gradually increased ( $7 \mathrm{~d}$ : $136.36 \pm 17.64$ vs. $202.20 \pm 29.26, p<0.05$; 14 d: $398.05 \pm 89.45$ vs. $464.49 \pm 51.25$; both $p<0.05$ ).

Higher Water Content Was Observed in the Brain of Rats Exposed to Hyperoxia One day after hyperoxic exposure, the water content of brain tissue in the hyperoxic group was significantly higher compared to the normoxic group $(65.26 \pm 3.20,61.34 \pm 2.21, p<0.05)$. After $7 \mathrm{~d}$ and $14 \mathrm{~d}$, the difference gradually increased $(7 \mathrm{~d}: 78.87 \pm 2.22 v s$. $74.76 \pm 2.99 ; 14 \mathrm{~d}: 78.32 \pm 2.43$ vs. $74.33 \pm 2.10$; both $p<0.05)$ (Fig. 1b).

Analysis of Brain Histopathological Results of Rats in Each Group HE staining showed that in the air group, neurons in the cerebral cortex were arranged in neat order, with regular cell morphology, large and round nuclei, with rare presence of inflammatory cells and cell edema. In the hyperoxic group, after 1-d exposure, edema and rupture of many cells occurred in the hippocampal area. In addition, partial nuclear dissolution and pyknosis, and few inflammatory cells were also observed.

The arrangement of nerve cells in cerebral cortex and hippocampus in the group that was exposed high oxygen for $7 \mathrm{~d}$; cell edema and rupture were increased in the group that was exposed to high oxygen for $1 \mathrm{~d}$, and the number of nuclear lysis, nuclear pyknosis and inflammatory cells were increased in the group that was exposed to high oxygen for $1 \mathrm{~d}$

After $14 \mathrm{~d}$ of high oxygen, the above reported changes were more obvious. The arrangement of nerve cells in the cerebral cortex and hippocampus was obviously disordered, the nucleus was relatively large, and the nuclear pyknosis was 
a
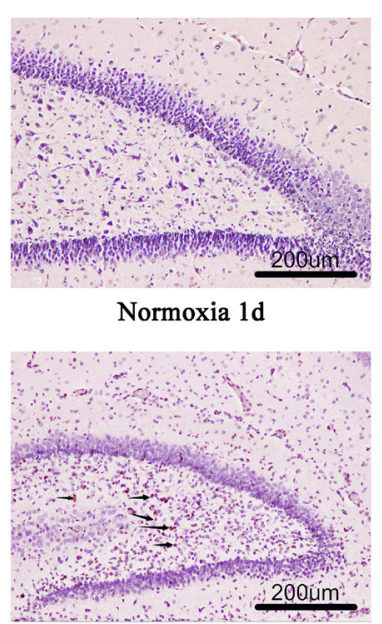

Hyperoxia 1d

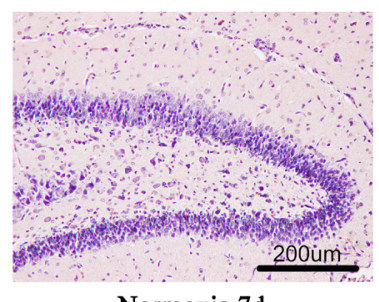

Normoxia $7 \mathrm{~d}$

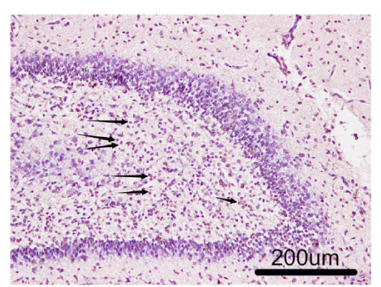

Hyperoxia 7d
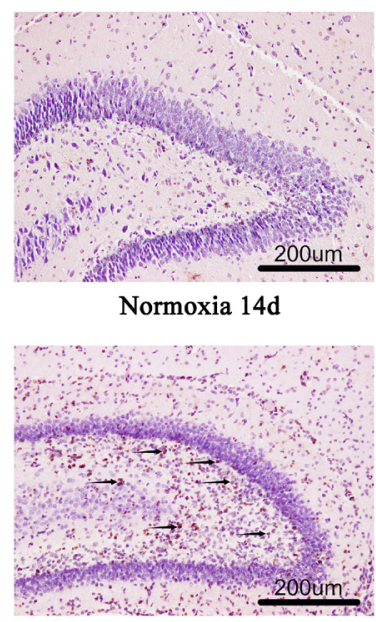

Hyperoxia 14d

b

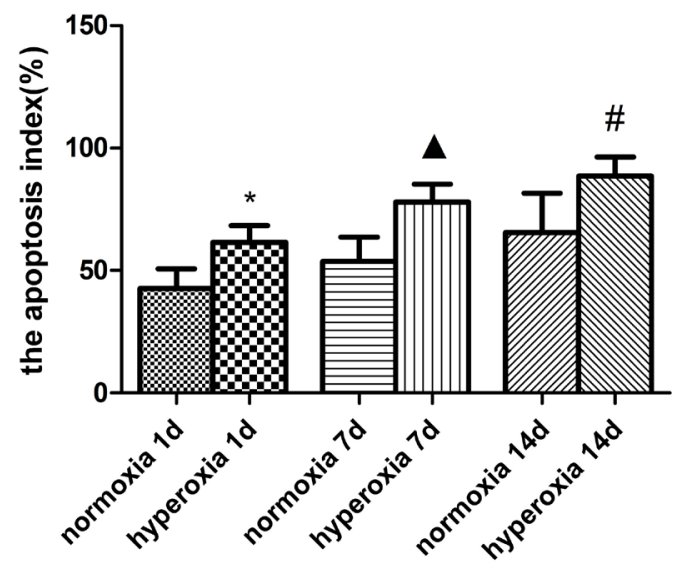

Fig. 3. (a): Tunel Stain in the Brain Tissues at Day 1, Day 7 and Day 14 in the Normoxic Group and the Hyperoxic Group (Tunel $\times 200$ ); (b): The Apoptosis Index at 1, 7 and 14 Days in Each Group

*Compared with normoxia 1d, $\boldsymbol{\Lambda}$ Compared with normoxia 7d, \# Compared with normoxia 14d, $p<0.05$, with statistical significance. (Color figure can be accessed in the online version.)

significantly increased. A hyperplasia was observed in few fibrous tissues. (Fig. 2)

Brain Tissue Apoptosis Index The apoptosis index of hippocampal nerve cells increased in hyperoxic group compared with the normoxic group at 1,7 and $14 \mathrm{~d}$, respectively. In Tunel staining, the nuclei of apoptotic cells were stained yellowish brown or brown. Apoptotic cells could be observed in the hippocampal area after $1 \mathrm{~d}$ of hyperoxic exposure while more apoptotic cells could be found in the group of hyperoxia on 7 and $14 \mathrm{~d}$. (Figs. 3a, 3b)

Results of Brain Tissue Reactive Oxygen Species in Each Group Compared with the normoxic group, the ROS gradually increased overtime in rats exposed to hyperoxia $(p<0.05)$. The content of reactive oxygen species in newborn rat brain tissue was increased in the group of hyperoxia 1d $(6446.58 \pm 574.36$, vs. 4953.43 \pm 422.16, $p<0.05)$ compared with the normoxia. The content of ROS in newborn rat brain tissue was also increased $(10324.77 \pm 886.51 \mathrm{vs}$. $9088.90 \pm 764.59, p<0.05)$ after $7 d$ exposure to hyperoxia. Compared with the 14-d normoxic group, the content of ROS increased (12086.84 \pm 1442.14 vs. 9696.58 $\pm 1174.62, p<0.05)$ too. All data are shown in Fig. 4.

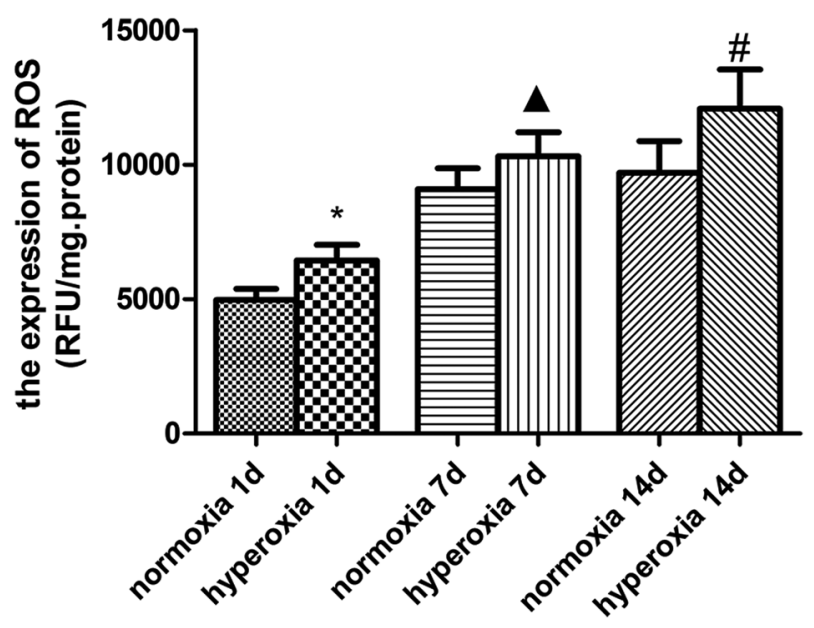

Fig. 4. Changes in the Expression of ROS in the Brain Tissue in Each Group

*Compared with normoxia 1d, $\boldsymbol{\Delta}$ Compared with normoxia 7d, \# Compared with normoxia $14 \mathrm{~d}, p<0.05$, with statistical significance. 


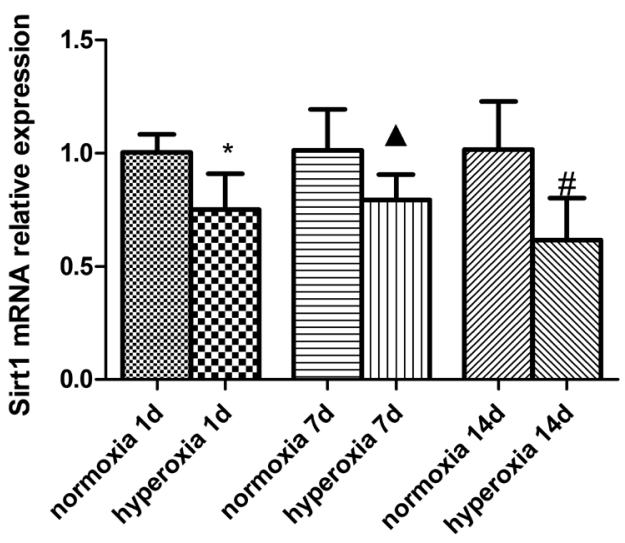

a

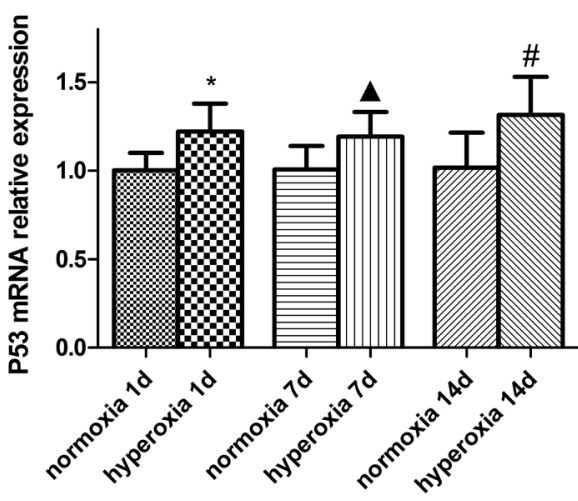

b

Fig. 5. (a): The Relative Expression of Sirt1 mRNA at 1, 7 and 14 Days in Each Group; (b): Changes in the Relative Expression of p53 mRNA in the Brain Tissue in Each Group

*Compared with normoxia 1d, $\boldsymbol{\Delta}$ Compared with normoxia 7d, \# Compared with normoxia 14d, $p<0.05$, with statistical significance.

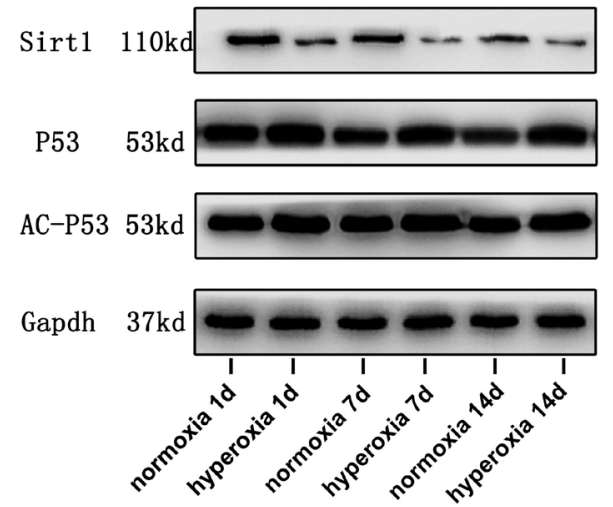

a

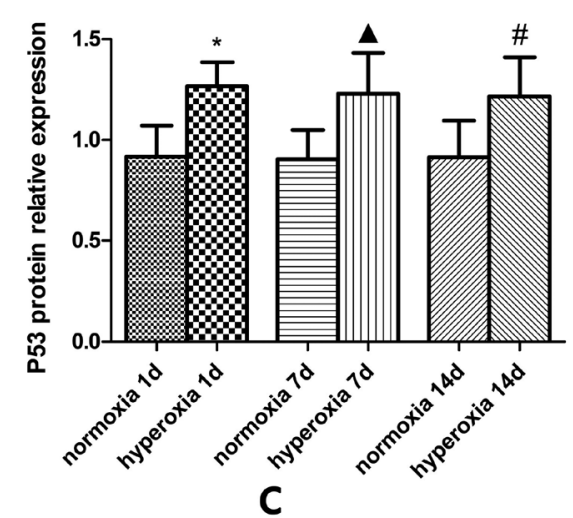

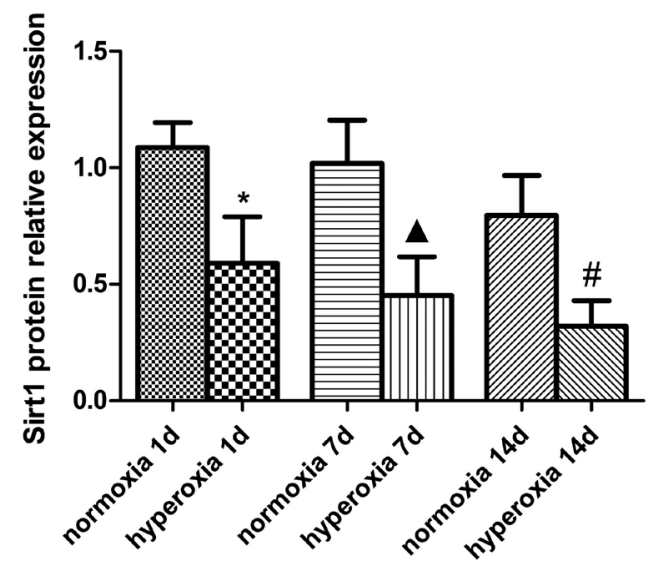

b

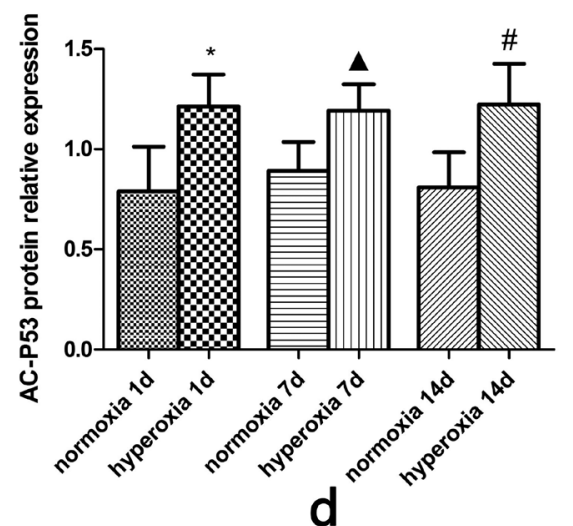

Fig. 6. (a): The Relative Expressions of Sirt1, p53, AC-p53 and Gapdh Protein at 1, 7 and 14 Days in Each Group; (b): Changes in the Relative Expression of Sirtl Protein in the Brain Tissue in Each Group

*Compared with normoxia 1d, $\mathbf{\Delta}$ Compared with normoxia $7 \mathrm{~d}$, \# Compared with normoxia 14d, $p<0.05$, with statistical significance. (c): Changes in the relative expression of p53 protein in the brain tissue in each group, ${ }^{*}$ Compared with normoxia $1 \mathrm{~d}$, $\boldsymbol{\Delta}$ Compared with normoxia $7 \mathrm{~d}$, \# Compared with normoxia $14 \mathrm{~d}$, $p<0.05$, with statistical significance. (d): Changes in the relative expression of AC-p53 protein in the brain tissue in each group (*Compared with normoxia 1d, $\boldsymbol{\Delta}$ Compared with normoxia 7d, \# Compared with normoxia 14d, $p<0.05$, with statistical significance).

The Relative Expression of Sirt1 and 553 in Normoxia and Hyperoxia Group Compared with the normoxia group, a gradual decrease of Sirt1 mRNA was observed in the brain tissue of newborn rats exposed to hyperoxia. The relative expression of Sirtl was decreased $(0.752 \pm 0.158,1.003 \pm 0.082$, $p<0.05)$ in the hyperoxic group of $1 \mathrm{~d}$ compared with the normoxic group. And at the time of exposure to hyperoxia for $7 \mathrm{~d}$, the relative expression of Sirt1 was declined in hyperoxic group compared with the normoxic group $(0.796 \pm 0.109$, $1.013 \pm 0.183, p<0.05)$. After exposing to hyperoxia for $14 \mathrm{~d}$, the hyperoxic and normoxic group showed the same trend with the relative expression data $0.619 \pm 0.183$ and $1.018 \pm 0.212$, 
respectively $(p<0.05)$. All data are shown in Fig. 5a.

Contrary, the relative expression of p53 mRNA gradually increased in rats exposed to hyperoxia compared to the normoxia group $(p<0.05)$. All data are shown in Fig. 5 b.

The Relative Expression of Sirt1, p53 and Acetylated p53 Protein in Normoxia and Hyperoxia Group The western blots were consistent with the RT-PCR results. Briefly, gradual decrease of Sirt1 protein was observed in the brain tissue of newborn rats exposed to hyperoxia $(p<0.05)$ (Figs. 6a, 6b); while the protein expression of p53 (Figs. 6a, 6c) and acetylated p53 (Figs. 6a, 6d) gradually increased in rats exposed to hyperoxia compared to the normoxia group $(p<0.05)$.

\section{DISCUSSION}

Many factors have been associated with the brain injury of prematurely born infants, such as preterm birth, perinatal infection, nutritional imbalance, and oxygen exposure. Most premature infants are born with hypoxemia, and with respiratory system disease, such as neonatal respiratory distress syndrome. Oxygen therapy can significantly improve the premature infant hypoxic condition. Nevertheless, considering that these patients have an undeveloped immune system, the target organs such as brain and lung are sensitive to damage factors. Deuber and Terhaar ${ }^{7)}$ have investigated the hyperoxia in prematurely born infants with gestational age that was less than 28 weeks, and found that blood oxygen saturation was greater than $92 \%$, which indicated that hyperoxic state was clearly associated with brain injury. ${ }^{7)}$ Furthermore, Semple et al. ${ }^{8)}$ have discovered that the brain development of newborn SD rats was similar to that of human newborns. Dzietko et al. ${ }^{9)}$ have established a hyperoxic brain injury model by exposing $6 \mathrm{~d}$ old newborn Wistar rats to $80 \%$ oxygen concentration, which they used to prove that hyperoxia increases the death of brain cells. In this study, we established a hyperoxic brain injury model in newborn SD rats and compared them with animals exposed to normoxia conditions. Our data showed a significantly reduced body weight in the hyperoxic group compared to the normoxic group, which was consistent with the findings of Jing et al. ${ }^{10)}$ In addition, we found that water content of brain in neonatal SD rats were increasing after exposing to hyperoxia, suggesting the occurrence of cerebral edema, which were consistent with the observation of H\&E staining: cell edema and rupture, partial nuclear dissolution and pyknosis, and infiltration of inflammatory cells. These phenomena further proved that the experimental model was successful and was consistent with the literature. ${ }^{6}$

Oxidative stress indicates an imbalance between the generation of free radicals and the resistance to oxidative substances, which in turn result in tissue damages. ${ }^{11)}$ Many studies have shown that oxidative stress has an important role in the occurrence and development of brain injury, considering that preterm newborns exhibit higher levels of oxidative stressdue to the inability to face the oxygen rich environment in which they are born into. During this condition, the body cannot effectively remove the oxidative stress reaction that produces reactive oxygen species, and leads to apoptosis. ${ }^{3,12)}$ The production of a large number of reactive oxygen species can lead to decreased Sirtl activity expression in the body ${ }^{13)}$ and increased apoptosis. SIRTs is a histone deacetylation enzyme, which has seven kinds of mammals homologue (Sirt1-Sirt7). Sirtl is widely expressed in human tissues, especially in the brain tissue. ${ }^{14)}$ Preclinical studies have shown that Sirtl has a protective role during the development of the brain. ${ }^{15-17)}$ For example, in astrocytes, Srit1 has a neuroprotective role through its antioxidant and anti-inflammatory functions. These effects are mainly mediated by the deacetylation of Sirt1 that regulates various key targets, such as FOXO, Ku70, $\mathrm{p} 53$, nuclear factor-kappaB $(\mathrm{NF}-\kappa \mathrm{B})$, etc. Li and Wang ${ }^{18)}$ have found that in sh-sy5y cells of neurons with Sirtl gene knockout, cell proliferation and differentiation functions are inhibited, which in turn is related to the increased expression of p53. Carloni et al. ${ }^{19)}$ have found that, with the increase of Sirt1 expression and activity, the expression of p53 and the acetylation of p53 decrease, and the neuroprotective effect increases. Wang et al. $^{20)}$ have also found that in animal models of epileptic seizures, the level of Sirtl decreases and the level of acetylated p53 is up-regulated, which in turn induces neuron loss and apoptosis in epileptic model rats.

In this study, the content of reactive oxygen species at 1,7 and $14 \mathrm{~d}$ after hyperoxia exposure was higher than that at the corresponding time points in the normoxic group, suggesting that the increase of reactive oxygen species in brain tissue was more obvious with the extension of hyperoxic exposure time; these data were consistent with the study of Vidal-Jorge. ${ }^{21)}$ In addition, after exposure to high oxygen, Sirtl mRNA expression and Sirtl protein expression also decreased, while the gene and protein expression level remained constant, p53 mRNA and p53 protein expression as well as acetylated p53 protein expression and cell apoptosis increased, which was consistent with other literature research. This suggested that high oxygen exposure can lead to brain injury in newborn SD rats, characterized by the increase of reactive oxygen species in brain tissue, decreased expression of Sirt1, increased p53 and acetylated p53, causing apoptosis. That is to say, the Sirt1 pathway, which decreases the deacetylation of $\mathrm{p} 53$, thus causing cell apoptosis may mediate the mechanism underlying brain injury induced by hyperxia.

\section{CONCLUSION}

Hyperoxic exposure can lead to brain injury in newborn SD rats. These events might be regulated by the Sirt1 pathway, which downregulates the deacetylation of $\mathrm{p} 53$, thereby increasing apoptosis.

Acknowledgments The work was supported by the National Natural Science Foundation of China [Grant number 81571480].

Conflict of Interest The authors declare no conflict of interest.

\section{REFERENCES}

1) Howson CP, Kinney MV, McDougall L, Lawn JE. Born too soon: preterm birth matters. Reprod. Health, 10 (Suppl. 1), S1 (2013).

2) Panfoli I, Candiano G, Malova M, De Angelis L, Cardiello V, Buonocore G, Ramenghi LA. Oxidative stress as a primary risk factor for brain damage in preterm newborns. Front Pediatr., 6, 369 (2018). 
3) Ozsurekci Y, Aykac K. Oxidative stress related diseases in newborns. Oxid. Med. Cell. Longev., 2016, 2768365 (2016).

4) Fujita Y, Yamashita T. Sirtuins in neuroendocrine regulation and neurological diseases. Front Neurosci., 12, 778 (2018).

5) Ong ALC, Ramasamy TS. Role of Sirtuin1-p53 regulatory axis in aging, cancer and cellular reprogramming. Ageing Res. Rev., 43, 64-80 (2018)

6) Kim YE, Park WS, Sung DK, Ahn SY, Sung SI, Yoo HS, Chang YS. Intratracheal transplantation of mesenchymal stem cells simultaneously attenuates both lung and brain injuries in hyperoxic newborn rats. Pediatr. Res., 80, 415-424 (2016).

7) Deuber C, Terhaar M. Hyperoxia in very preterm infants: a systematic review of the literature. J. Perinat. Neonatal Nurs., 25, 268-274 (2011)

8) Semple BD, Blomgren K, Gimlin K, Ferriero DM, Noble-Haeusslein LJ. Brain development in rodents and humans: Identifying benchmarks of maturation and vulnerability to injury across species. Prog. Neurobiol., 106-107, 1-16 (2013).

9) Dzietko M, Boos V, Sifringer M, Polley O, Gerstner B, Genz K, Endesfelder S, Borner C, Jacotot E, Chauvier D, Obladen M, Buhrer C, Felderhoff-Mueser U. A critical role for Fas/CD-95 dependent signaling pathways in the pathogenesis of hyperoxia-induced brain injury. Ann. Neurol., 64, 664-673 (2008).

10) Jing X, Huang YW, Jarzembowski J, Shi Y, Konduri GG, Teng RJ. Caffeine ameliorates hyperoxia-induced lung injury by protecting GCH1 function in neonatal rat pups. Pediatr. Res., 82, 483-489 (2017).

11) Czerska M, Mikolajewska K, Zielinski M, Gromadzinska J, Wasowicz W. Today's oxidative stress markers. Med. Pr., 66, 393-405 (2015).

12) Thibeault DW. The precarious antioxidant defenses of the preterm infant. Am. J. Perinatol., 17, 167-182 (2000).

13) Song SB, Hwang ES. A rise in ATP, ROS, and mitochondrial content upon glucose withdrawal correlates with a dysregulated mitochondria turnover mediated by the activation of the protein deacetylase SIRT1. Cells, 8, 11 (2018).

14) Sakamoto J, Miura T, Shimamoto K, Horio Y. Predominant expression of Sir2alpha, an NAD-dependent histone deacetylase, in the embryonic mouse heart and brain. FEBS Lett., 556, 281-286 (2004).

15) Ramadori G, Fujikawa T, Fukuda M, Anderson J, Morgan DA, Mostoslavsky R, Stuart RC, Perello M, Vianna CR, Nillni EA, Rahmouni K, Coppari R. SIRT1 deacetylase in POMC neurons is required for homeostatic defenses against diet-induced obesity. Cell Metab., 12, 78-87 (2010).

16) Guarente L. Sirtuins, aging, and metabolism. Cold Spring Harb. Symp. Quant. Biol., 76, 81-90 (2011).

17) Cheng Y, Takeuchi H, Sonobe Y, Jin S, Wang Y, Horiuchi H, Parajuli B, Kawanokuchi J, Mizuno T, Suzumura A. Sirtuin 1 attenuates oxidative stress via upregulation of superoxide dismutase 2 and catalase in astrocytes. J. Neuroimmunol., 269, 38-43 (2014).

18) Li H, Wang R. Blocking SIRT1 inhibits cell proliferation and promotes aging through the PI3K/AKT pathway. Life Sci., 190, 84-90 (2017)

19) Carloni S, Riparini G, Buonocore G, Balduini W. Rapid modulation of the silent information regulator 1 by melatonin after hypoxiaischemia in the neonatal rat brain. J. Pineal Res., 63, e12434 (2017)

20) Wang D, Li Z, Zhang Y, Wang G, Wei M, Hu Y, Ma S, Jiang Y, Che N, Wang X, Yao J, Yin J. Targeting of microRNA-199a-5p protects against pilocarpine-induced status epilepticus and seizure damage via SIRT1-p53 cascade. Epilepsia, 57, 706-716 (2016).

21) Vidal-Jorge M, Sanchez-Guerrero A, Mur-Bonet G, Castro L, Radoi A, Riveiro M, Fernandez-Prado N, Baena J, Poca MA, Sahuquillo J. Does normobaric hyperoxia cause oxidative stress in the injured brain? A microdialysis study using 8-iso-prostaglandin F2alpha as a biomarker. J. Neurotrauma., 34, 2731-2742 (2017). 